\title{
Variation Iteration Method for The Approximate Solution of Nonlinear Burgers Equation
}

\author{
1E.J. Mamadu ${ }^{1}$ and ${ }^{2 *}$ I.N. Njoseh \\ 1 Department of Mathematics, University of Ilorin, P.M.B 1515, Ilorin, Nigeria \\ 2Department of Mathematics, Abraka, Delta State University, Abraka, Nigeria. \\ [Corresponding author: e-mail: njoseh@delsu.edu.ng, E : 08035786279]
}

\section{ABSTRACT}

In this study, we considered the numerical solution of the nonlinear Burgers equation using the Variational Iteration Method (VIM). The method seeks to examine the convergence of solutions of the Burgers equation at the expense of the parameters $x$ and $t$, of which the amount of errors depends. Numerical experimentation was carried out on the Burgers equation with the Variational Iteration Method (VIM). The resulting solution showed that the rate of convergence decreases with increase in the values of the parameters $x$ and $t$ at each iterate level. However, as the number of iterations increases, there is a rapid rate of convergence of the approximate solution to the analytic solution. Results obtained with the Variational Iteration Method (VIM) on the Burgers equation were compared with the exact found in literature. All computational framework of the research were performed with the aid of Maple 18 software.

keywords: Variational Iteration Method, Burgers Equation, Partial Differential Equations, Approximate Solution, Mean Value Theorem, Schwartz Inequality.

\section{INTRODUCTION}

The study of the Burgers' equation is an important aspect of compressible and incompressible fluid models and the critical analysis of these models have been triggered by many researchers in recent years for understanding the basic principles of a class of physical flows and for examining various computational procedures. The Burgers equation has relevant applications in many field of Mathematics which include, hydrodynamic, gas dynamic, time-space stochastic processes, rocket motor, acoustic, number theory, heat conduction, shock waves, etc. (Burger, 1948). Hence, obtaining the exact resolution of this equation for a precise analysis of models under consideration is of great significance.

However, available analytic methods are insufficient in handling these equations due to large computational and round-off errors which arise due to linearization and perturbation. Thus, approximate methods have become more relevant as developed by researchers over the years to effectively handle these problems.
These include: the Variational Iteration Method (VIM) (He, 1998), the Reconstruction of Variation Iteration Method (RVIM) (Esfandyaripour, 2013), Homotopy Analysis Method (HAM) (Molabahrami and Khani, 2009), the Homotopy Perturbation Method (He, 2004 and 2005), the piecewiseadaptive decomposition method (Ramos, 2008) etc.

The Variational Iteration Method (VIM) was first proposed by the Chinese mathematician, J.H. He in He (1998) for solving both linear and nonlinear problems. The method involves the construction of a correction functional for the problem in question from which the Lagrange multiplier is derived optimally using the Variational Theory. This method has been extended to solve different classes of problems by various researchers such as, Fredholm and Volterra integro-differential equations (Mamadu and Njoseh, 2016; Wazwaz, 2011; Abdelkhani, 1993; Abbasbandy and Shivanian, 2009), partial differential equations (Duangpithak, 2012; He, 1998; He, 1999), delay differential equations (Liu et al., 2013), fractional diffusion equations (Gao 


\section{Nigerian Journal of Basic and Applied Science (June, 2016, 24(1): 70-75}

et al., 2016), etc. Njoseh and Mamadu (2017) equally coupled the Variational Iteration Method and the Homotopy Perturbation Method in a modified sense for the numerical treatment of the Burgers equation.

In this paper, the Variation Iteration Method (VIM) is initiated to solve nonlinear partial differential equation of which the Burgers equation is a special kind. For this Burgers equation, the initial approximation is freely chosen so as to satisfy the analytic solution. The method gives the solution in a more compact rapidly convergent series. For numerical illustration, the method was applied to onedimensional nonlinear Burgers equation of the form $\frac{\partial y}{\partial t}+y \frac{\partial y}{\partial x}=\frac{\partial^{2} y}{d x^{2}}$
Subject to the initial condition:

$$
y(x, t)=\frac{1}{2}-\frac{1}{2} \tanh \left(\frac{x}{4}\right) \text {. }
$$

\section{METHODOLOGY}

\section{Basic Ideas of VIM}

Consider the general differential equation

$L y(x, t)+N y(x, t)=f(x, t)$

with prescribed auxiliary conditions, where $L$ is a linear operator of the highest derivative, $N$ a non linear term, $y(x, t)$ is unknown function and $f(x, t)$ is a source term.

Using VIM (He, 1999; Duangpithak, 2012; Esfandyaripour et al., 2013), we can construct correction functional for equation (2) as:

$$
y_{k+1}(x, t)=y_{k}(x, t)+\int_{0}^{x} \lambda(\xi)\left[L y_{k}(x, \xi)+N \widetilde{y}_{k}(x, \xi)-f(, x \xi)\right] d \xi, k \geq 0
$$

where $\lambda$ is a general Lagrange multiplier, $\tilde{y}_{k}(x, t)=0$, i.e, $\tilde{y}_{k}(x, t)$ is a restricted variable, where it can be identified via variational theory (He, 1999, He and $\mathrm{Wu}, 2006)$. However, the Lagrange multiplier can still be obtained using the formula proposed by Abbasbandy and Shivanian (2009)

$\lambda(s)=\frac{(-1)^{n}(s-x)^{n}}{(n-1) !}$,

Where $n$ is the order of the derivative.

\section{Variation Iteration Method for the Burgers Equation}

To apply the Variation Iteration Method (VIM), we redefine equation (2) as

$L y(x, t)+N y(x, t)=f(x, t)$

where

$L y(x, t)=\frac{\partial^{2} y}{\partial x^{2}}, \quad N y(x, t)=$

$y \frac{\partial y}{\partial x}, f(x, t)=\frac{\partial y}{\partial t}$.

By the theory of Variational Iteration Method, we construct a correction functional for equation (4) as follows:

$$
y_{k+1}(x, t)=y_{k}(x, t)+\int_{0}^{t} \lambda(s)\left[\frac{\partial^{2} y_{k}(x, s)}{\partial x^{2}}-y_{k}(x, s) \frac{\partial y_{k}(x, s)}{\partial x}-\frac{\partial y_{k}(x, s)}{\partial t}\right] d s, k \geq 0
$$

and to obtain the Lagrange multiplier $\lambda(s)$, we $\quad y_{k}(x, s)$, bearing in mind that the variation of take the variation of (5) with respect to $\tilde{y}_{k}(x, t)$ is zero, as:

$$
\delta y_{k+1}(x, t)=\delta y_{k}(x, t)+\delta \int_{0}^{t} \lambda(s)\left[\frac{\partial^{2} y_{k}(x, s)}{\partial x^{2}}-y_{k}(x, s) \frac{\partial y_{k}(x, s)}{\partial x}-\frac{\partial y_{k}(x, s)}{\partial t}\right] d s, \quad k \geq 0 .
$$

$\delta y_{k+1}(x, t)=$

$\delta y_{k}(x, t)+\delta\left[\lambda(s)\left(\frac{\partial y_{k}(x, s)}{\partial t}-y_{k}(x, s)\right)\right]_{s=t}-\int_{0}^{t} \lambda^{\prime}(s)\left(\frac{\partial y_{k}(x, s)}{\partial t}-y_{k}(x, s)\right) d s, \quad k \geq 0$

Hence, equation (7) provides the stationary conditions

$\lambda(s)-1=0$ 
Mamadu and Njoseh: Variation Iteration Method for the Approximate Solution of Nonlinear Burgers Equation

$\left[\lambda^{\prime}(s)\right]_{s=t}=0, \quad\left[\lambda^{\prime \prime}(s)\right]_{s=t}=0 \quad(9)$

Equation (8) is the Lagrange multiplier and We now rewrite Equation (5) as

Equation (9) is the boundary condition.

$$
y_{k+1}(x, t)=y_{k}(x, t)-\int_{0}^{t}\left[\frac{\partial^{2} y_{k}(x, s)}{\partial x^{2}}-y_{k}(x, s) \frac{\partial y_{k}(x, s)}{\partial x}-\frac{\partial y_{k}(x, s)}{\partial t}\right] d s, \quad k \geq 0
$$

Equation (10) is the Variation Iteration Method for the Burgers equation (1).

\section{Convergence Analysis of the Method}

Let $H=\left(\alpha_{0}, \alpha_{1}\right) \times[0, T], \quad \mathrm{H}$ is a Hilbert in equation (1) such that space, and define

$y: H \rightarrow R, \int_{H} y^{2}(x) d x d w<+\infty$.

\section{Theorem 1.}

Let

$\frac{\partial y}{\partial t}=V(y)$
$V(y)=\frac{\partial^{2} y}{\partial x^{2}}-y \frac{\partial y}{\partial x}$

then the variational iteration method of equation (1) converges if;

i. $(V(y)-V(Y), y-Y) \geq \alpha\|y-Y\|^{2}, \alpha>0, y, Y \in H$.

ii. For $w>0$, there exist $I(w)>0$ for all $\|y\| \leq w,\|Y\| \leq w, \quad y, Y \in H$, then

\section{Proof}

$$
(V(y)-V(Y), y-Y) \geq I(w)\|y-Y\|\|r\|, \quad r \in H .
$$

Let $y, Y \in H$, then for $\alpha>0$, we have

$$
(V(y)-V(Y), y-Y)=\left(\left(\frac{\partial^{2} y}{\partial x^{2}}-y \frac{\partial y}{\partial x}\right)-\left(\frac{\partial^{2} Y}{\partial x^{2}}-Y \frac{\partial Y}{\partial x}\right), y-Y\right) .
$$

Applying the Schwartz inequality, we have

where

$$
\begin{gathered}
(V(y)-V(Y), y-Y) \leq \alpha_{1}\left\|\frac{\partial^{2} y}{\partial x^{2}}-y \frac{\partial y}{\partial x}-\frac{\partial^{2} Y}{\partial x^{2}}+Y \frac{\partial Y}{\partial x}\right\|\|y-Y\| \\
\leq \alpha_{1}\left\|\frac{\partial^{2}}{\partial x^{2}}(y-Y)+(y-Y) \frac{\partial}{\partial x}(Y-y)\right\|\|y-Y\| \\
\leq \alpha_{1}\left\|\frac{\partial^{2}}{\partial x^{2}} e(x)+e(x) \frac{\partial}{\partial x}(-e(x))\right\|\|e(x)\|
\end{gathered}
$$

$$
e(x)=y(x)-Y(x)
$$

$y(x)$ is the computed solution, and $Y(x)$ is the By the mean value theorem, we have that exact solution.

$$
(V(y)-V(Y), e(x)) \geq \frac{1}{4} \alpha_{1} w^{2}\|e(x)\|^{2} .
$$

Also, if $I(w)>0$ for all $\|y\| \leq w,\|Y\| \leq w, \quad y, Y \in H$, then we have

This completes the proof.

$$
\begin{aligned}
&(V(y)-V(Y), e(x))=\left(\frac{\partial^{2}}{\partial x^{2}} e(x)+e(x) \frac{\partial}{\partial x}(-e(x)), e(x)\right) \\
& \geq I(w)\|e(x)\|\|r\|, r \in H .
\end{aligned}
$$




\section{Nigerian Journal of Basic and Applied Science (June, 2016, 24(1): 70-75}

Theorem 2.

The Burgers equation as defined in equation (1) converges rapidly to the exact for $t=\frac{\tau}{10^{m}}, \tau>$ 0 , and $n$ is the number of iterations.

(Readers are referred to Mamadu and Njoseh (2017) for the proof of Theorem 2.)

\section{Numerical Illustrations}

We consider the one dimensional nonlinear Burgers equation for illustration in order to show the effectiveness and reliability of the method with the aid of Maple 18 software for our computations. The results obtained are compared with the exact solution available in literature. Given the Burgers equation $\frac{\partial y}{\partial t}+y \frac{\partial y}{\partial x}=\frac{\partial^{2} y}{\partial x^{2}}$

subject to the initial condition:

$y(x, t)=\frac{1}{2}-\frac{1}{2} \tanh \left(\frac{x}{4}\right)$.

The analytic solution is

$y(x, t)=\frac{1}{2}-\frac{1}{2} \tanh \left(\frac{1}{4}\left(x-\frac{t}{2}\right)\right)$.

By imposing the initial condition on the exact solution, we take the initial approximation as

$y_{0}(x, s)=\frac{1}{2}-\frac{1}{2} \tanh \left(\frac{x}{4}\right)$.

Hence, for $k=0$ in Equation (10), we have

$$
y_{1}=\frac{1}{2}-\frac{1}{2} \tanh \left(\frac{1}{4} x\right)+\frac{1}{4} \tanh \left(\frac{1}{4} x\right)\left(\frac{1}{4}-\frac{1}{4} \tanh \left(\frac{1}{4} x\right)^{2}\right) t-\left(\frac{1}{2}-\frac{1}{2} \tanh \left(\frac{1}{4} x\right)\right)\left(-\frac{1}{8}+\frac{1}{8} \frac{1}{4} \tanh \left(\frac{1}{4} x\right)^{2}\right) t
$$

The Tables given below show the amount of absolute error for $y_{1}, y_{2}$ and $y_{4}$ and the amount of error responses to the parameters $x$ and $t$. . Here, $x$ ranges from 0.1 to 1.0 , and $t$ ranges from 0.2 to 3.0 .

\section{DISCUSSION OF RESULTS}

Apparently from Tables 1 to 3 , it is obvious that the amount of errors responses to the parameters $x$ and $t$ indicates that the convergence of the method as applied to the Burgers equation decreases with increase in the values of the parameters $x$ and $t$. It is also noted that the rate of convergence of the scheme improve adequately as the number of iterations increases. Thus, by theorem 2, $(n+1)$ iterations will see the approximate solution converging absolutely to the analytic solution.

Table 1: Computation of absolute errors for first approximation, $y_{1}$

\begin{tabular}{|c|ccccccccc|}
\hline \multirow{2}{*}{$\mathrm{X}$} & \multicolumn{7}{|c|}{ First approximation, $\boldsymbol{y}_{\mathbf{1}}$} \\
\cline { 2 - 10 } & $t=0.2$ & $t=0.6$ & $t=0.9$ & $t=1.2$ & $t=1.5$ & $t=1.7$ & $t=2.0$ & $t=2.5$ & $t=3.0$ \\
\hline 0.1 & $5.2058 \mathrm{e}-06$ & $1.1700 \mathrm{e}-08$ & $7.8775 \mathrm{e}-05$ & $2.7925 \mathrm{e}-04$ & $6.5173 \mathrm{e}-04$ & $1.0196 \mathrm{e}-03$ & $1.7853 \mathrm{e}-03$ & $3.7389 \mathrm{e}-03$ & $6.6977 \mathrm{e}-03$ \\
0.2 & $1.2989 \mathrm{e}-05$ & $7.0178 \mathrm{e}-05$ & $7.8952 \mathrm{e}-05$ & $3.7280 \mathrm{e}-07$ & $2.1684 \mathrm{e}-04$ & $4.6332 \mathrm{e}-04$ & $1.0212 \mathrm{e}-03$ & $2.5646 \mathrm{e}-03$ & $5.0424 \mathrm{e}-03$ \\
0.3 & $2.0708 \mathrm{e}-05$ & $1.3999 \mathrm{e}-04$ & $2.3628 \mathrm{e}-04$ & $2.7999 \mathrm{e}-04$ & $2.1911 \mathrm{e}-04$ & $9.5251 \mathrm{e}-05$ & $2.5209 \mathrm{e}-04$ & $1.3780 \mathrm{e}-03$ & $3.3631 \mathrm{e}-03$ \\
0.4 & $2.0323 \mathrm{e}-05$ & $2.0911 \mathrm{e}-04$ & $3.9244 \mathrm{e}-04$ & $5.5821 \mathrm{e}-04$ & $6.5397 \mathrm{e}-04$ & $6.5334 \mathrm{e}-04$ & $5.1823 \mathrm{e}-04$ & $1.8478 \mathrm{e}-04$ & $1.0678 \mathrm{e}-03$ \\
0.5 & $3.5800 \mathrm{e}-05$ & $2.7720 \mathrm{e}-04$ & $5.4666 \mathrm{e}-04$ & $8.3367 \mathrm{e}-04$ & $1.0856 \mathrm{e}-03$ & $1.2082 \mathrm{e}-03$ & $1.2860 \mathrm{e}-03$ & $1.0093 \mathrm{e}-03$ & $3.5270 \mathrm{e}-05$ \\
0.6 & $4.3100 \mathrm{e}-05$ & $3.4392 \mathrm{e}-04$ & $6.9818 \mathrm{e}-04$ & $1.1050 \mathrm{e}-03$ & $1.5119 \mathrm{e}-03$ & $1.7571 \mathrm{e}-03$ & $2.0474 \mathrm{e}-03$ & $2.1984 \mathrm{e}-03$ & $1.7380 \mathrm{e}-03$ \\
0.7 & $5.0192 \mathrm{e}-05$ & $4.0897 \mathrm{e}-04$ & $0.4629 \mathrm{e}-04$ & $1.3710 \mathrm{e}-03$ & $1.9307 \mathrm{e}-03$ & $2.2974 \mathrm{e}-03$ & $2.7987 \mathrm{e}-03$ & $3.3767 \mathrm{e}-03$ & $3.4322 \mathrm{e}-03$ \\
0.8 & $5.7044 \mathrm{e}-05$ & $4.7205 \mathrm{e}-04$ & $9.9031 \mathrm{e}-04$ & $1.6302 \mathrm{e}-03$ & $2.3402 \mathrm{e}-03$ & $2.8265 \mathrm{e}-03$ & $3.5365 \mathrm{e}-03$ & $4.5386 \mathrm{e}-03$ & $5.1097 \mathrm{e}-03$ \\
0.9 & $6.3626 \mathrm{e}-05$ & $5.3287 \mathrm{e}-04$ & $1.1296 \mathrm{e}-03$ & $1.0817 \mathrm{e}-03$ & $2.7384 \mathrm{e}-03$ & $3.3420 \mathrm{e}-03$ & $4.2572 \mathrm{e}-03$ & $5.6786 \mathrm{e}-03$ & $6.7625 \mathrm{e}-03$ \\
1.0 & $6.9912 \mathrm{e}-05$ & $5.9120 \mathrm{e}-04$ & $1.2635 \mathrm{e}-03$ & $2.1242 \mathrm{e}-03$ & $3.1236 \mathrm{e}-03$ & $3.8415 \mathrm{e}-03$ & $4.9575 \mathrm{e}-03$ & $6.7914 \mathrm{e}-03$ & $8.3030 \mathrm{e}-03$ \\
\hline
\end{tabular}


Table 2: Computation of absolute errors for second approximation, $y_{2}$.

\begin{tabular}{|c|ccccccccc|}
\hline \multirow{2}{*}{$\mathrm{X}$} & \multicolumn{7}{|c|}{ Second Approximation, $\boldsymbol{y}_{\mathbf{2}}$} \\
\cline { 2 - 10 } & $t=0.2$ & $t=0.6$ & $t=0.9$ & $t=1.2$ & $t=1.5$ & $t=1.7$ & $t=2.0$ & $t=2.5$ & $t=3.0$ \\
\hline 0.1 & $2.7302 \mathrm{e}-06$ & $7.3753 \mathrm{e}-05$ & $2.4869 \mathrm{e}-04$ & $5.8035 \mathrm{e}-04$ & $1.1457 \mathrm{e}-03$ & $1.6635 \mathrm{e}-03$ & $2.6959 \mathrm{e}-03$ & $5.2125 \mathrm{e}-03$ & $0.8929 \mathrm{e}-03$ \\
0.2 & $2.8429 \mathrm{e}-06$ & $7.6970 \mathrm{e}-05$ & $2.5999 \mathrm{e}-04$ & $6.1618 \mathrm{e}-04$ & $1.2021 \mathrm{e}-03$ & $1.7475 \mathrm{e}-03$ & $2.8374 \mathrm{e}-03$ & $5.5036 \mathrm{e}-03$ & $9.4201 \mathrm{e}-03$ \\
0.3 & $2.9406 \mathrm{e}-06$ & $7.9778 \mathrm{e}-05$ & $2.6991 \mathrm{e}-04$ & $6.4074 \mathrm{e}-04$ & $1.2521 \mathrm{e}-03$ & $1.8222 \mathrm{e}-03$ & $2.9639 \mathrm{e}-03$ & $5.7658 \mathrm{e}-03$ & $9.8984 \mathrm{e}-03$ \\
0.4 & $3.0221 \mathrm{e}-06$ & $8.2155 \mathrm{e}-05$ & $2.7837 \mathrm{e}-04$ & $6.6182 \mathrm{e}-04$ & $1.2953 \mathrm{e}-03$ & $1.8071 \mathrm{e}-03$ & $3.0743 \mathrm{e}-03$ & $5.9969 \mathrm{e}-03$ & $1.0323 \mathrm{e}-02$ \\
0.5 & $3.0876 \mathrm{e}-06$ & $8.4084 \mathrm{e}-05$ & $2.8530 \mathrm{e}-04$ & $6.7927 \mathrm{e}-04$ & $1.3313 \mathrm{e}-03$ & $1.9416 \mathrm{e}-03$ & $3.1678 \mathrm{e}-03$ & $6.1949 \mathrm{e}-03$ & $1.0692 \mathrm{e}-02$ \\
0.6 & $3.1364 \mathrm{e}-06$ & $8.5551 \mathrm{e}-05$ & $2.9066 \mathrm{e}-04$ & $6.9296 \mathrm{e}-04$ & $1.3600 \mathrm{e}-03$ & $1.9853 \mathrm{e}-03$ & $3.2436 \mathrm{e}-03$ & $6.3584 \mathrm{e}-03$ & $1.1001 \mathrm{e}-02$ \\
0.7 & $3.1679 \mathrm{e}-06$ & $8.6551 \mathrm{e}-05$ & $2.9442 \mathrm{e}-04$ & $7.0281 \mathrm{e}-04$ & $1.3812 \mathrm{e}-03$ & $2.0179 \mathrm{e}-03$ & $3.3013 \mathrm{e}-03$ & $6.4863 \mathrm{e}-03$ & $1.1240 \mathrm{e}-02$ \\
0.0 & $3.1827 \mathrm{e}-06$ & $8.7081 \mathrm{e}-05$ & $2.9657 \mathrm{e}-04$ & $7.0800 \mathrm{e}-04$ & $1.3947 \mathrm{e}-03$ & $2.0393 \mathrm{e}-03$ & $3.3407 \mathrm{e}-03$ & $6.5779 \mathrm{e}-03$ & $1.1432 \mathrm{e}-02$ \\
0.9 & $3.1802 \mathrm{e}-06$ & $8.7144 \mathrm{e}-05$ & $2.9713 \mathrm{e}-04$ & $7.1095 \mathrm{e}-04$ & $1.4006 \mathrm{e}-03$ & $2.0496 \mathrm{e}-03$ & $3.3616 \mathrm{e}-03$ & $6.6329 \mathrm{e}-03$ & $1.1552 \mathrm{e}-02$ \\
1.0 & $3.1617 \mathrm{e}-06$ & $8.6752 \mathrm{e}-05$ & $2.9611 \mathrm{e}-04$ & $7.0930 \mathrm{e}-04$ & $1.3989 \mathrm{e}-03$ & $2.0408 \mathrm{e}-03$ & $3.3643 \mathrm{e}-03$ & $6.6517 \mathrm{e}-03$ & $1.1609 \mathrm{e}-02$ \\
\hline
\end{tabular}

Table 3: Computation of absolute errors for fourth approximation, $y_{4}$.

\begin{tabular}{|c|ccccccccc|}
\hline \multirow{2}{*}{$\mathrm{X}$} & \multicolumn{7}{|c|}{ Second Approximation, $\boldsymbol{y}_{\mathbf{4}}$} \\
\cline { 2 - 10 } & $t=0.2$ & $t=0.6$ & $t=0.9$ & $t=1.2$ & $t=1.5$ & $t=1.7$ & $t=2.0$ & $t=2.5$ & $t=3.0$ \\
\hline 0.1 & $2.6000 \mathrm{e}-09$ & $6.5500 \mathrm{e}-07$ & $5.1128 \mathrm{e}-06$ & $2.2166 \mathrm{e}-05$ & $6.9608 \mathrm{e}-05$ & $1.3267 \mathrm{e}-04$ & $3.0771 \mathrm{e}-04$ & $9.8519 \mathrm{e}-04$ & $2.5716 \mathrm{e}-03$ \\
0.2 & $2.7000 \mathrm{e}-09$ & $6.2530 \mathrm{e}-07$ & $4.8782 \mathrm{e}-06$ & $2.1116 \mathrm{e}-05$ & $6.6211 \mathrm{e}-05$ & $1.2608 \mathrm{e}-04$ & $2.9211 \mathrm{e}-04$ & $9.3394 \mathrm{e}-04$ & $2.4360 \mathrm{e}-03$ \\
0.3 & $2.4000 \mathrm{e}-09$ & $5.8650 \mathrm{e}-07$ & $4.5601 \mathrm{e}-06$ & $1.9696 \mathrm{e}-05$ & $6.1650 \mathrm{e}-05$ & $1.1727 \mathrm{e}-04$ & $2.7132 \mathrm{e}-04$ & $8.6595 \mathrm{e}-04$ & $2.2563 \mathrm{e}-03$ \\
0.4 & $2.3000 \mathrm{e}-09$ & $5.3660 \mathrm{e}-07$ & $4.1616 \mathrm{e}-06$ & $1.7931 \mathrm{e}-05$ & $5.6001 \mathrm{e}-05$ & $1.0630 \mathrm{e}-04$ & $2.4570 \mathrm{e}-04$ & $7.0237 \mathrm{e}-04$ & $2.0355 \mathrm{e}-03$ \\
0.5 & $2.0000 \mathrm{e}-09$ & $4.7760 \mathrm{e}-07$ & $3.6907 \mathrm{e}-06$ & $1.5851 \mathrm{e}-05$ & $4.9367 \mathrm{e}-05$ & $9.3620 \mathrm{e}-05$ & $2.1572 \mathrm{e}-04$ & $6.8401 \mathrm{e}-04$ & $1.7779 \mathrm{e}-03$ \\
0.6 & $2.0000 \mathrm{e}-09$ & $4.1000 \mathrm{e}-07$ & $3.1565 \mathrm{e}-06$ & $1.3500 \mathrm{e}-05$ & $4.1804 \mathrm{e}-05$ & $7.9244 \mathrm{e}-05$ & $1.0202 \mathrm{e}-04$ & $5.7530 \mathrm{e}-04$ & $1.4890 \mathrm{e}-03$ \\
0.7 & $1.4000 \mathrm{e}-09$ & $3.3640 \mathrm{e}-07$ & $2.5693 \mathrm{e}-06$ & $1.0926 \mathrm{e}-05$ & $3.3709 \mathrm{e}-05$ & $6.3557 \mathrm{e}-05$ & $1.4529 \mathrm{e}-04$ & $4.5619 \mathrm{e}-04$ & $1.1749 \mathrm{e}-03$ \\
0.8 & $8.0000 \mathrm{e}-10$ & $2.5630 \mathrm{e}-07$ & $1.9431 \mathrm{e}-06$ & $8.1823 \mathrm{e}-06$ & $2.5016 \mathrm{e}-05$ & $4.6897 \mathrm{e}-05$ & $1.0635 \mathrm{e}-04$ & $3.3010 \mathrm{e}-04$ & $8.4274 \mathrm{e}-04$ \\
0.9 & $7.0000 \mathrm{e}-10$ & $1.7400 \mathrm{e}-07$ & $1.2901 \mathrm{e}-06$ & $5.3299 \mathrm{e}-06$ & $1.5994 \mathrm{e}-05$ & $2.9627 \mathrm{e}-05$ & $6.6042 \mathrm{e}-05$ & $1.9981 \mathrm{e}-04$ & $4.9982 \mathrm{e}-04$ \\
1.0 & $6.0000 \mathrm{e}-10$ & $8.9100 \mathrm{e}-00$ & $6.2510 \mathrm{e}-07$ & $2.4295 \mathrm{e}-06$ & $6.8383 \mathrm{e}-06$ & $1.2118 \mathrm{e}-05$ & $2.5234 \mathrm{e}-05$ & $6.8155 \mathrm{e}-05$ & $1.5371 \mathrm{e}-04$ \\
\hline
\end{tabular}

\section{CONCLUSION}

This paper has considered the Variational Iteration Method for solving the Burgers equations. The method is simple and straightforward with no hidden or weak

\section{REFERENCES}

Abbasbandy, S. and Shivanian, E. (2009). Application of the Variational Iteration Method for System of Nonlinear Volterra's Integro-differential equations. Mathematics and Computational Applications, 14(2):147-158.

Abdelkhani, J. (1993). Higher order methods for solving Volterra Integro-differential Equations of the First Kind. Appl. Math. and Comput., 58(1):97-101. assumptions. The results obtained in this research with VIM on Burgers equations agree with results in Esfandyaripour et al. (2013). All computational analysis was performed with the computer application software, Maple 18.

Burger J.M. (1948). A mathematical model illustrating the theory of turbulence. Adv. Appl. Math. 1:171-199.

Duangpithak, S. (2012).Variation Iteration Method for Special Nonlinear Partial Differential Equations. Int. Journal of Math. Analysis, 6(22):1071-1077.

Esfandyaripour, E., Hadi, M., Esfandyaripour, M., Hossein, M.J., Doabsari, S.M.E. and Ganji, D.D. ( 2013). Approximate Solution to Burgers Equation using 


\section{Nigerian Journal of Basic and Applied Science (June, 2016, 24(1): 70-75}

Reconstruction of Variation Method. Applied Mathematics, 3(2):45-49.

Gao, F., Srivastava, H.M., Gao, Y. and Yang, X. (2016). A Coupling Method involving the Sumudu Transform and the Variational Iteration Method for a class of Local Fractional Diffusion Equations. Journal of Nonlinear Science and Application, 9:5830-5835.

He, J.H. (1998). Variational Iteration Approach to Nonlinear Problems and its Applications. Mech. Appl., 20:30-31.

He, J.H. (1999). Variation Iteration Method: A Kind of Nonlinear Analytical Technique: Some Example. International Journal of nonlinear Mechanics, 34(4): 699-708

He, J.H. (2004). Homotopy Perturbation Method for Nonlinear Oscillators with Discontinuities. Applied Mathematics and Computations,151(1):287-292

He, J.H. (2005). Homotopy Perturbation Method for Bifurcation on Nonlinear Problems. International Journal of Nonlinear Sciences and Numerical Stimulation, 6(2):207-208.

$\mathrm{He}$, J.H. and Wu, X.H. (2006). Construction of Solitary Solution and Compaction-like Solution by Variation Iteration Method. Chaos Soliton and Fract., 29(1):108-113.

Liu, H., Xiao, A., and Su. L. (2013).Convergence of Variational Iteration Method for Second Delay Differential Equation. Journal of Applied Mathematics, 9 pages. Article ID:634670

Mamadu, E.J. and Njoseh, I.N. (2016). On the Convergence Variational Iteration Method for the Numerical Solution of Nonlinear Integro-differential Equations. Transactions of Nigeria Association of Mathematical Physic, 2: 65-70.

Molabahrami, A. and Khani, F. (2009).The Homotopy Analysis Method to solve the Burgers-Huxley Equation. Nonlinear Anal. RWA., 10(2):589-600.

Njoseh, I.N. and Mamadu, E.J. (2017). Modified Variational Iteration Homotopy Perturbation Method for the Approximate solution of Burgers Equation. Transactions of the Nigeria Association of Mathematical Physics, 3:1-4.

Noor, M.A. and Mohyud-Din , S.T. (2009). Variation Iteration Method for Unsteady Flow of Gas through a Porous Medium using He's Polynomials and Pade Approximants. Comput. Math, wth Appl., 58:2182-2189.

Ramos, J.I. (2008). Piecewise-Adaptive Decomposition Methods. Chaos Soliton and Fract. 40(4):1623-1636.

Wazwaz, A.M. (2011). Linear and nonlinear integral equations: methods and applications, Springer, Berlin. 